7-13 per cent; palmitoleic acid, $13-20$ per cent; oleic and linoleic acids, 18-33 per cent; unsaturated acids containing 20 carbon atoms, $19-32$ per cent; unsaturated acids containing 22 carbon atoms, 10-19 per cent. The depth of the yellow colour of the oil was found to be roughly proportional to its vitamin potency.

The variations in potency of different samples are not apparently directly dependent on the age or sexual condition of the fish, but on the character and amount of the food eaten, which varies according to the season. On the other hand, examination of the fish and several crustacea which form the food supply of the cod showed them to be singularly devoid of both vitamins. It has been shown that phytoplankton synthesise vitamin A (Ahmad, Biochem. Jour., vol. 24 , p. $860 ; 1930)$ : zooplankton contain none and neither contain appreciable quantities of vitamin $\mathrm{D}$, although there may be small amounts present in zooplankton. There is no evidence that the cod can synthesise either, though the possibility cannot be excluded. The alternative is that the fish retains the small amounts in its food in its liver, so that over a course of months with a plentiful food supply this organ comes to contain considerable amounts. The potency of the oil varies inversely with the amount obtained from the liver: prior to spawning there is a utilisation of the liver fat, especially in the female; but the vitamin store is not proportionally depleted, so that the oil has a high potency. The authors believe this to be the explanation of the high vitamin content of Newfoundland oil.

From an examination of the effects of different methods of manufacture upon the palatability, keeping properties, and potency of the oil, the following procedure is recommended: Livers should be fresh and steamed immediately they have been removed from the fish. Steam at 60-100 lb. pressure per sq. in. gives better results than steam at a lower pressure, and the process should not be too short. Subsequent refining should be restricted to removal of moisture, debris, and stearine by chilling and centrifuging or pressure ; finally, medicinal oils should be stored in vessels impermeable to light and containing as little free air space as possible. Deterioration is caused by the activity of the liver enzymes; hence the necessity for using only fresh material and for adequate steam. ing to destroy them. High pressure steam also gives a higher yield of oil. It was found that the replacement of air in the storage casks by an inert gas was difficult to carry out properly and did not increase the keeping properties of the oil. Protection from light and undue exposure to air is, however, of great importance.

As a result of their work, the authors put forward the following specifications for medicinal cod liver oil : The colour, when measured in a 1-in. cell, should not be greater than 10 yellow Lovibond units and 0.5 red unit. The free fatty acid (as oleic acid) should not be greater than 0.5 per cent, preferably below 0.3 per cent. The unsaponifiable matter should not be more than 1.5 per cent, preferably below 1 per cent. The vitamin A colour test should give a higher value than 7 blue Lovibond units, when carried out by the authors' technique. For oil intended for farm-stock, the colour and free acidity may be slightly greater but the amount of unsaponifiable matter and vitamin A present should be the same. There appears to be no doubt that oils from Scotland and, especially, Newfoundland are capable of meeting these specifications.

\title{
Mosquito Control.*
}

THE Report for 1930 of the British Mosquito Control Institute, Hayling Island, Hampshire, is a record of two and a half years' work, the previous Report having been presented in June 1928. The Institute, it may be added, was built and equipped in 1925 by Mr. John F. Marshall, who has since occupied the position as director without remuneration. It was incorporated by licence of the Board of Trade in February 1927 and, by a deed executed at the same time, the building and its equipment were leased to trustees for a term of years. No financial aid is received from any official funds, and the income of the Institute is derived solely from the results of its own activities and from subscriptions and donations. Funds are greatly needed in order to enable the work to become more self-supporting, since the present income falls a long way short of covering expenditure.

The activities of the Institute have markedly increased since the issue of the last Report, and the interest shown by the outside public in this work is borne out by the fact that more than 1400 visitors (scientific and others) inspected the museum and laboratory during 1930. Advisory work relating to mosquitoes, their identification and control, is carried out by means of inspection and by correspondence. Inspections are generally undertaken by the director's assistant at a pre-arranged charge which includes the submission of $a$ report and the recommendation of remedial measures. Various lines of investigation have been carried out during the period under review, including tests of fly-killing preparations and of cresol. containing larvicides, at the request of commercial

* British Mosquito Control Institute. Report of the Director. Pre* British Mosquito Control Institute. Report of the Director. Pre30 illustrations. Hayling Island, Hampshire. firms. A study has also been commenced with regard to the breeding of arboreal mosquitoes in cavities (natural and artificial) in sawn-off parts of trees of different species.

In June 1929 a special malaria course, arranged in connexion with the League of Nations and the London School of Tropical Medicine, included in its programme a three-day visit to the Institute for laboratory and field instruction. A number of medical officers took part in this work. In September 1930 a week's course, of a tentative character, on mosquito research for university students was given, and was sufficiently appreciated to warrant arrangements being made for its repetition during the present year. Among other educational activities, the director has delivered lectures on mosquito control to various institutions, etc., while demonstrations have been conducted at a number of seientific meetings, exhibitions, etc. By way of technical apparatus, the advances made in connexion with photomicrography and stereograph methods are described in the Report. Mention needs also to be made of the small but growing library of the Institute: a number of new books have been added both by gift and by purchase, while certain of the more important periodicals are taken in regularly. The director of the Institute is to be congratulated upon the initiative and energy he is giving to the development of his charge and on the progress so far achieved.

Scientific societies, educational bodies, local authorities, and other associations desirous of supporting this useful work may become 'collective' members. Members (whether individual or collective) subscribe one guinea per annum and receive a copy of the publications of the Institute. 